\title{
Restrictions over two-dimensional gauge models with Thirring-like interaction.
}

\author{
D. Dalmazi ${ }^{b *}$ and A. de Souza Dutra ${ }^{a, b \dagger}$ \\ ${ }^{a}$ Abdus Salam ICTP, Strada Costiera 11, 34014 Trieste Italy \\ ${ }^{b}$ UNESP - Campus de Guaratinguetá - DFQ ${ }^{\ddagger}$ \\ Av. Dr. Ariberto Pereira da Cunha, 333 \\ 12516-410 Guaratinguetá SP Brasil
}

December 18, 2018

\begin{abstract}
Some years ago, it was shown how fermion self-interacting terms of the Thirring type impact the usual structure of massless two-dimensional gauge theories [1]. In that work only the cases of pure vector and pure chiral gauge couplings have been considered and the corresponding Thirring term was also pure vector and pure chiral respectively, such that the vector (or chiral) Schwinger model should not lose its chirality structure due to the addition of the quartic interaction term. Here we extend this analysis to a generalized vector and axial coupling both for the gauge interaction and the quartic fermionic interactions. The idea is to perform quantization without losing the original structure of the gauge coupling. In order to do that we make use of an arbitrariness in the definition of the Thirring-like interaction.
\end{abstract}

\footnotetext{
${ }^{*}$ E-mail: dalmazi@feg.unesp.br

†E-mail: dutra@feg.unesp.br

${ }^{\ddagger}$ Permanent Institution
} 
The physics of the two-dimensional quantum field theories has a long and beautiful history. Particularly, one of the most important and beautiful features of this restricted number of dimensions is that it allows the exact solvability of some important fermionic models, which is intrinsically connected with the idea of bosonization. The bosonization program leads to a deeper understanding of the internal structure of the models. People have been trying to extend it for higher dimensions for a long time. One of the first models to be exactly solved in the history of the quantum field theory is the Thirring model [2], and a little time after that Schwinger presented to the world what is now a landmark for the bosonization technique, the so called Schwinger model [3], whose solution through operator approach was carried out by Lowenstein and Swieca in another beautiful and classical paper [4]. Its path integral solution was obtained in [5] by following an approach introduced by Fujikawa [6]. After several works analyzing a number of properties and applications of these and other exact two-dimensional systems, Jackiw and Rajaraman [7] published a seminal paper in this subject where they have presented the first consistent model with an anomalously broken gauge symmetry, the chiral Schwinger model (CSM). In a non-trivial extension, some authors developed these ideas for the case of the arbitrary left and right couplings which may be called a generalized Schwinger model (GSM) [8, 9, 10].

Some years ago [1], some of the above ingredients were combined together in order to explore the effect of the quartic fermionic self-interaction of the Thirring type on, among other physical properties, the dynamically generated photon mass. It has been shown in that work that, for instance in the case of the Schwinger model, the dynamically generated photon mass is screened due to the self-interacting Thirring term as follows:

$$
m^{2}=\frac{e^{2}}{\pi+g^{2}},
$$

We can also speak of a kind of duality, in the sense that a model with strong Thirring coupling $g$ can be traded in another one with small electromagnetic coupling $e$ [1].

In this work we initially extend the analysis of the impact of fermionic self-interactions on the structure of the gauge theories in $1+1$ dimensions and, after demonstrating that the special case of CSM can not be taken as a limit of the general model, we present the difficulties in performing this task without losing the chirality of the original CSM model. 


\section{Generalized Schwinger model with Thirring coupling}

Our starting point is a generalized Schwinger model, below $P_{ \pm}=\left(1 \pm \gamma_{5}\right) / 2$, :

$$
\mathcal{L}=\bar{\Psi}\left(i \not \partial+e_{R} \gamma^{\mu} P_{+} A_{\mu}+e_{L} \gamma^{\mu} P_{-} A_{\mu}\right) \Psi-\frac{1}{4} F^{\mu \nu} F_{\mu \nu}
$$

Next we would like to add a general quartic fermionic interaction such that it is both chiral and gauge invariant while keeping the explicit Poincare covariance. Before we introduce our model it is important for our purposes to make use of a two dimensional spinor identity proved in [11], namely,

$$
(\bar{\Psi} M \Psi)^{2}=\operatorname{det} M(\bar{\Psi} \Psi)^{2} \quad
$$

Where $M$ may be any $2 \times 2$ nonsingular matrix. Now, it follows from $\operatorname{det} P_{ \pm}=0$ and (3) that $\left(\bar{\Psi} \gamma_{\mu} P_{ \pm} \Psi\right)^{2}=0$. Consequently, by making use of the representations $\bar{\Psi} \gamma_{\mu} \Psi=\bar{\Psi} \gamma_{\mu}\left(P_{+}+P_{-}\right) \Psi$ and $\bar{\Psi} \gamma_{\mu} \gamma_{5} \Psi=\bar{\Psi} \gamma_{\mu}\left(P_{+}-P_{-}\right) \Psi$ we can derive

$$
a\left(\bar{\Psi} \gamma_{\mu} \Psi\right)^{2}+b\left(\bar{\Psi} \gamma_{\mu} \gamma_{5} \Psi\right)^{2}+c\left(\bar{\Psi} \gamma_{\mu} \Psi\right)\left(\bar{\Psi} \gamma^{\mu} \gamma_{5} \Psi\right)=\frac{(a-b)}{e_{R} e_{L}}\left[\bar{\Psi} \gamma_{\mu}\left(e_{R} P_{+}+e_{L} P_{-}\right) \Psi\right]^{2}
$$

Where the constants $a, b, c$ are arbitrary and we have assumed $e_{R}, e_{L} \neq 0$. Note that the right handed side of (4) is independent of $c$ which stems from the fact that the term $j^{\mu} j_{\mu}^{5}$ which multiplies $c$ vanishes identically by virtue of (3) and the afore mentioned representations. The vanishing of both sides of (41) for $a=b$ can be easily checked from (3) and $\operatorname{det} \gamma_{5}=-1$.

Therefore, with the exception of the chiral Schwinger models $e_{R}=0$ or $e_{L}=0$, the identity (44) allows us to add a rather general quartic fermionic term to the generalized Schwinger model in a such way that the chiral structure of the gauge coupling is maintained. That is,

$$
\begin{aligned}
\mathcal{L} & =\bar{\Psi}\left(i \not \partial+e_{R} \gamma^{\mu} P_{+} A_{\mu}+e_{L} \gamma^{\mu} P_{-} A_{\mu}\right) \Psi-\frac{g^{2}}{2}\left(\bar{\Psi} \gamma_{\mu}\left(e_{R} P_{+}+e_{L} P_{-}\right) \Psi\right)^{2} \\
& -\frac{1}{4} F^{\mu \nu} F_{\mu \nu}+j^{\mu} \bar{\Psi} \gamma_{\mu}\left(e_{R} P_{+}+e_{L} P_{-}\right) \Psi
\end{aligned}
$$


where we have introduced a source term for future use. In order to deal with this model, one usually reduces the order of the quartic interaction by means of an auxiliary vector field $B_{\mu}$ which leads us to a physically equivalent effective theory

$$
\begin{aligned}
\mathcal{L}_{e f f} & =\bar{\Psi}\left(i \not \partial+e_{R} \gamma^{\mu} P_{+} A_{\mu}+e_{L} \gamma^{\mu} P_{-} A_{\mu}\right) \Psi-g B_{\mu} \bar{\Psi}\left(e_{R} \gamma^{\mu} P_{+}+e_{L} \gamma^{\mu} P_{-}\right) \Psi+\frac{1}{2} B_{\mu} B^{\mu} \\
& -\frac{1}{4} F^{\mu \nu} F_{\mu \nu}+j^{\mu} \bar{\Psi} \gamma_{\mu}\left(e_{R} P_{+}+e_{L} P_{-}\right) \Psi
\end{aligned}
$$

By integrating over $B_{\mu}$ in the path integral or using its equations of motion we recover (5)). Now it is clear that the fermionic fields interact with the gauge field, the auxiliary field and the source through the combination:

$$
C_{\mu} \equiv A_{\mu}+j_{\mu}-g B_{\mu}
$$

in terms of which, the Lagrangian density can be rewritten as

$$
\mathcal{L}_{e f f}=\bar{\Psi}\left(i \not \partial+e_{R} \gamma^{\mu} P_{+} C_{\mu}+e_{L} \gamma^{\mu} P_{-} C_{\mu}\right) \Psi+\frac{1}{2 g^{2}}\left(A_{\mu}+j_{\mu}-C_{\mu}\right)^{2}-\frac{1}{4} F^{\mu \nu} F_{\mu \nu}
$$

This last expression can be bosonized through standard techniques as in [10] with the following steps: First of all, one introduces a general vector decomposition $C_{\mu}=\epsilon_{\mu \nu} \partial^{\nu} \chi+\partial_{\mu} \eta$. Next, one decouples the fermionic fields through a chiral and a gauge transformation:

$$
\Psi=\exp \left[i e_{V}\left(\eta+\chi \gamma_{5}\right)+i e_{A}\left(\eta \gamma_{5}-\chi\right)\right] \zeta
$$

where

$$
e_{V}=e_{R}+e_{L} \quad ; \quad e_{A}=e_{R}-e_{L}
$$

The chiral transformation has a nontrivial Jacobian [6]. The effective theory including the Jacobian becomes

$$
\mathcal{L}_{e f f}=\bar{\zeta}(i \not \partial) \zeta+\frac{1}{2} \chi \square^{2} \chi+\frac{1}{2 \pi}\left(\frac{e_{A}^{2}}{4}-m^{2}\right) \eta \square \eta+
$$




$$
\begin{aligned}
& \frac{1}{2 \pi}\left(\frac{e_{V}^{2}}{4}+m^{2}\right) \chi \square \chi-\frac{e_{V} e_{A}}{4 \pi} \eta \square \chi+ \\
& +\frac{1}{2 g^{2}}\left(A_{\mu}+j_{\mu}-\epsilon_{\mu \nu} \partial^{\nu} \chi-\partial_{\mu} \eta\right)^{2}-\frac{1}{4} F^{\mu \nu} F_{\mu \nu}
\end{aligned}
$$

where $m$ is an arbitrary parameter with mass dimension. The terms which depend on $\chi$ and $\eta$ are combined back into the field $C_{\mu}$. Some of them can be written as a functional integral over a scalar field $\phi$. After all those steps we obtain the intermediate effective Lagrangian density,

$$
\begin{aligned}
& \mathcal{L}_{e f f}=\bar{\zeta}(i \not \partial) \zeta+\frac{1}{2}\left(\partial_{\mu} \phi\right)^{2}-\frac{1}{2 \sqrt{\pi}}\left[e_{A} g^{\mu \nu}-e_{V} \epsilon^{\mu \nu}\right] \partial_{\nu} \phi C_{\mu}+ \\
& \frac{m^{2}}{2 \pi} C_{\mu}^{2}+\frac{1}{2 g^{2}}\left(A_{\mu}+j_{\mu}-C_{\mu}\right)^{2}-\frac{1}{4} F^{\mu \nu} F_{\mu \nu},
\end{aligned}
$$

At this point we can change back the variables from the field $C_{\mu}$ to $B_{\mu}$ according to (7). Integrating out the auxiliary field $B_{\mu}$ and the free Dirac field $\zeta$ we obtain a full bosonized Lagrangian density generalizing the work of [1]:

$$
\begin{aligned}
\mathcal{L}_{\text {bosonic }} & =\frac{1}{2}\left[1+\frac{e_{R} e_{L} g^{2}}{\pi+m^{2} g^{2}}\right]\left(\partial_{\mu} \phi\right)^{2}-\frac{\sqrt{\pi}\left[e_{A} g^{\mu \nu}-e_{V} \epsilon^{\mu \nu}\right] \partial_{\nu} \phi}{2\left(\pi+m^{2} g^{2}\right)}\left(A_{\mu}+j_{\mu}\right) \\
& +\frac{m^{2}}{2\left(\pi+m^{2} g^{2}\right)}\left(A_{\mu}+j_{\mu}\right)^{2}-\frac{1}{4} F^{\mu \nu} F_{\mu \nu} .
\end{aligned}
$$

Note that for $g=0$ the expression (13) at $j_{\mu}=0$ reproduces the result of [10] for the GSM.

Now we analyze the particle content of the bosonized theory. Integrating over the scalar field in the absence of external sources we obtain an effective nonlocal action for the photon:

$$
\mathcal{L}_{e f f}(A)=-\frac{1}{4} F^{\mu \nu}\left[1+\frac{\alpha\left(e_{V}^{2}+e_{A}^{2}\right)}{4 \square}\right] F_{\mu \nu}+\left(\frac{m^{2}}{K}-\frac{\alpha e_{A}^{2}}{4}\right) \frac{A_{\mu} A^{\mu}}{2}+\frac{\alpha e_{V} e_{A}}{4} \epsilon^{\mu \nu} \partial_{\mu} A_{\nu} \frac{1}{\square} \partial_{\beta} A^{\beta},
$$

where we have the constants:

$$
K=\pi+m^{2} g^{2} \quad ; \quad \alpha=\frac{\pi}{K\left(K+e_{R} e_{L} g^{2}\right)} .
$$

In the vector case $e_{R}=e_{L} \equiv e$ we can recover the gauge symmetry by fixing conveniently the arbitrary parameter $m^{2}=0$ which leads, redefining $g \rightarrow g / e$, to a nonlocal gauge theory with 
a massive photon $m_{p h}^{2}=e^{2} /\left(\pi+g^{2}\right)$ which reproduces the result of [1] and the result of the Schwinger model for $g=0$. In the general case the gauge symmetry is broken, so we can compute the propagator without gauge fixing. We first notice that $\mathcal{L}_{\text {eff }}(A)=A_{\mu} G^{\mu \nu} A_{\nu} / 2$ where the kinetic operator is of the form $G_{\mu \nu}=a g_{\mu \nu}+b \theta_{\mu \nu}+c V_{\mu \nu}$ with the definitions in momentum space $\theta_{\mu \nu}=g_{\mu \nu}-k_{\mu} k_{\nu} / k^{2}, V_{\mu \nu}=T_{\mu \nu}+T_{\nu \mu}$, with $T_{\mu \nu}=\epsilon_{\alpha \mu} k_{\nu} k^{\alpha}$. It is easy to check that the propagator is given by:

$$
\left\langle A_{\mu}(k) A_{\nu}(-k)\right\rangle=G_{\mu \nu}^{-1}=\frac{1}{D}\left[(a+b) g_{\mu \nu}-b \theta_{\mu \nu}-c V_{\mu \nu}\right]
$$

where

$$
\begin{aligned}
& a=\frac{m^{2}}{K}-\frac{\alpha e_{A}^{2}}{4} \quad ; \quad b=\frac{\alpha\left(e_{V}^{2}+e_{A}^{2}\right)}{4}-k^{2} \quad ; \quad c=\frac{\alpha e_{V} e_{A}}{4 k^{2}} \\
& D=(a+b) a+k^{4} c^{2}=k^{2}\left(\frac{\alpha e_{A}^{2}}{4}+\frac{m^{2}}{K}\right)-\frac{m^{2}}{K}\left[\alpha e_{R} e_{L}+\frac{m^{2}}{K}\right]
\end{aligned}
$$

The last term in the propagator (16) contains a pole at $k^{2}=0$. However, if we saturate the propagator with conserved currents and calculate the residue at this pole we have a vanishing result, i.e., $\lim _{k^{2} \rightarrow 0} k^{2} J_{\mu}\left\langle A^{\mu}(k) A^{\nu}(-k)\right\rangle J_{\nu}^{*}=0$. Therefore we do not have a propagating massless particle as one might think. On the other hand if we repeat this calculation at the pole coming from the condition $D=0$, we have a positive residue indicating a physical massive photon $k^{2}=m_{p h}^{2}$ whose mass depends on the arbitrary regularization parameter as follows:

$$
m_{p h}^{2}=\frac{m^{2}\left[m^{2}+\left(e_{V}^{2}-e_{A}^{2}\right) / 4\right]}{m^{2} g^{2}\left[m^{2}+\left(e_{V}^{2}-e_{A}^{2}\right) / 4\right]+\pi\left(m^{2}-e_{A}^{2} / 4\right)}
$$

The result (19) is both in agreement with the chiral case $\left(e_{V}=e_{A}\right)$ treated in [1] and the GSM without Thirring interaction $\left(g^{2}=0\right)$ studied in [12]. As in those cases, the arbitrary parameter $m^{2}$ must satisfy a specific bound in order to avoid tachyons.

Finally, we return to the issues of bosonization and the chiral limit. Since the quadratic term in the sources in (13) can only give rise to contact terms in the correlation functions of the $U(1)$ current, by comparing (13) with (5) we read off the bosonization rule: 


$$
\bar{\Psi} \gamma^{\mu}\left(e_{R} P_{+}+e_{L} P_{-}\right) \Psi=\bar{\Psi} \gamma^{\mu}\left(e_{V}+e_{A} \gamma_{5}\right) \Psi=\frac{m^{2}}{\left(\pi+m^{2} g^{2}\right)} A^{\mu}-\frac{\sqrt{\pi}\left[e_{A} g^{\mu \nu}-e_{V} \epsilon^{\mu \nu}\right] \partial_{\nu} \phi}{2\left(\pi+m^{2} g^{2}\right)}
$$

As in the chiral case studied in [1], we remark that the bosonization rule for the above generalized current is ambiguous due to the arbitrary, regularization dependent, parameter $m^{2}$. As commented in [1], we notice that the identity $\left(\bar{\Psi} \gamma^{\mu} P_{ \pm} \Psi\right)^{2}=0$ will not be respected by the bosonic map (20) in the chiral cases $e_{R}=0$ or $e_{L}=0$. This is a quantum effect, since it is caused by the gauge field term on the right handed side of (20) which appears due to the regularization procedure. In fact, had we started with the CSM plus a chiral Thirring interaction:

$$
\mathcal{L}=\bar{\Psi}\left[i \not \partial+e P_{ \pm} \gamma^{\mu}\left(A_{\mu}+j_{\mu}\right)\right] \Psi-\frac{1}{4} F^{\mu \nu} F_{\mu \nu}-\frac{g^{2}}{2}\left(\bar{\Psi} \gamma_{\mu}\left(P_{ \pm}\right) \Psi\right)^{2}
$$

since the last term vanishes identically, see comment after (3), we would end up with another bosonized Lagrangian density different from (13):

$$
\mathcal{L}_{\text {bosonic }}(a)=\frac{1}{2}\left(\partial_{\mu} \varphi\right)^{2}-\frac{e}{\sqrt{\pi}}\left(g^{\mu \nu} \pm \epsilon^{\mu \nu}\right) \partial_{\nu} \varphi\left(A_{\mu}+j_{\mu}\right)+\frac{a e^{2}}{2 \pi}\left(A_{\mu}+j_{\mu}\right)^{2}-\frac{1}{4} F_{\mu \nu}^{2},
$$

where $a$ is the Jackiw-Rajaraman [7] parameter which represents regularization ambiguities. Analogous to the derivation of (20) we derive from (21) and (22) a new bosonization rule which is also ambiguous but differs from (20), namely,

$$
e \bar{\Psi} \gamma^{\mu} P_{ \pm} \Psi(a)=\frac{a e^{2}}{\pi} A^{\mu}-\frac{e}{\sqrt{\pi}}\left(g^{\mu \nu} \pm \epsilon^{\mu \nu}\right) \partial_{\nu} \varphi
$$

There is of course no trace of the Thirring interaction in (22) and (23). On the other hand, in the chiral limits $\left(e_{R}, e_{L}\right) \rightarrow(0, e)$ or $\left(e_{R}, e_{L}\right) \rightarrow(e, 0)$, the Lagrangian density (5) coincides with (21) after the trivial replacement $g \rightarrow g e_{R}$ in (21). However, differently from (22) and (23) we have some dependance on the Thirring coupling constant in (13) and (20) in the chiral limits. Even if we redefine the arbitrary parameter $m^{2}$ such that $m^{2} /\left(\pi+m^{2} g^{2}\right) \rightarrow a e^{2}$ we still have a mismatch between formulas (13) and (22) and also (20) and (23). The difference might be interpreted as a finite charge renormalization. Furthermore, we should identify $\phi=\varphi$ for $e_{L}=0$ while for $e_{R}=0$ we have $\phi=-\varphi$. We can only make the couple of equations (13), (20) coincide with $(22),(23)$ 
in the chiral limit either by taking $g^{2} \rightarrow 0$ or selecting out the parameters $m^{2}=0=a$. As a consequence we should conclude that there is apparently no way of introducing the Thirring-like interaction for the CSM without losing its chiral characteristic. In fact, it can be shown that, for a large class of non-chiral Thirring-like interactions, the chirality of the original fermions is lost as it

should be expected. Finally, a quite interesting and intriguing conclusion from above is that one can introduce the Thirring-like interaction consistently, preserving the original mixture of right-left modes, except for the CSM models.

\section{Acknowledgments}

The authors are grateful to CNPq for partial financial support. This work has been finished during a visit (ASD) within the Associate Scheme of the Abdus Salam ICTP.

\section{References}

[1] A. de Souza Dutra, C. P. Natividade, H. Boschi-Filho, R. L. P. G. Amaral and L. V. Belvedere, Phys. Rev. D 55 (1997) 4931.

[2] W. Thirring, Ann. Phys. (N. Y.) 3 (1958) 3.

[3] J. Schwinger, Phys. Rev. 128 (1962) 2425.

[4] J. H. Lowenstein adn J. Swieca, Ann. Phys. (N. Y.) 68 (1971) 112.

[5] R. E. Gamboa-Saravi, F. A. Schaposnik and J. E. Solomin, Nucl. Phys. B 185 (1980) 239.

[6] K. Fujikawa, Phys. Rev. Lett. 42 (1979) 1195; Phys. Rev. D21 (1980) 2848.

[7] R. Jackiw and R. Rajaraman, Phys. Rev. Lett. 54 (1985) 1219.

[8] M. Chanowitz, Phys.Lett. B 171 (1986) 280.

[9] D. Boyanovsky, I. Schmidt and M. F. L. Goltterman,Ann. Phys. (N. Y.) 185 (1988). 
[10] A. de Souza Dutra, Int. J. Mod. Phys. A 9 (1994) 2229, and references therein.

[11] C. E. I. Carneiro, J. A. Mignaco and M. T. Thomaz, Phys. Rev. D 36 (1987) R1282.

[12] C. Naon and C. Wotzasek, J. of Phys. G 16 (1990)795. 